\title{
REMARQUES AU SUJET DU SARCOME A CYSTICERQUES DANS LE FOIE DU RAT
}

\author{
Par F. LARROUSSE \\ Agrégé des Facultés de médecine (Strasbourg)
}

Le sarcome du foie provoqué chez le rat par la larve du Tæria crassicollis, le Cysticercus fasciolaris représente, à mon avis, le cancer expérimental type, facile à réaliser dans certaines conditions. Dans beaucoup de laboratoires, on donne la préférence au cancer du goudron, qui a l'inconvénient d'avoir des données étiologiques plus incertaines et sujettes à de nombreuses controverses. Dans le sarcome du foie du rat, signalé pour la première fois par Borrel en 1906, le facteur étiologique ne fait aucun doute ; il s'agit d'un parasite, d'une larve de cestode. Il y a parasite, il y a sarcome avec, souvent, métastases ; le sarcome peut ètre greffé : c'est un cancer. Pour le provoquer et en faire l'étude, ainsi que l'ont démontré les deux auteurs américains Bullock et Curtis, il suffit d'avoir un chat qui héberge le Trnia crassicollis et des rats. Tous les rats qui ingèrent des œufs présentent des cysticerques dans le foie, mais tous ne font pas de sarcome ; certains élevages mème n'en font pas du tout. Alors que conclure ? C'est que le cysticerque à lui seul ne suffit pas, il faut autre chose. Deux hypothèses seulement peuvent être envisagées sérieusement.

La larve du Tænia apporte ou n'apporte pas un virus, le virus du sarcome, ou bien elle agit par ses toxines sur certains éléments cellulaires qui existent ou n'existent pas chez les rats envisagés. Il est difficile actuellement de prendre nettement position: les deux solutions sont possibles. Pour Borrel, l'hypothèse du virus est la plus vraisemblable, et je dois reconnaître que les arguments en faveur d'un germe infectieux apporté par la larve du Tænia sont nombreux. Doit-on rejeter pour cela l'hypothèse d'une action possible des toxines et poisons du ver sur certains éléments cellulaires ? Cela je ne le crois pas. Le but de cette note n'est pas de peser un à un les arguments en faveur des deux solutions possibles; les données sont encore trop incertaines et il serait imprudent de vouloir conclure. Je veux simplement relater deux faits qui paraissent avoir une certaine importance, que l'on 
peut interpréter suivant ses tendances personnelles, qui peuvent en tous cas servir de point de départ à des études expérimentales plus fécondes.

En collaboration avec le professeur Borrel, j'ai signalé (1) que le cysticerque pouvait être le vecteur du bacille de la lèpre murine et amener la localisation et la multiplication de ce dernier dans la membrane qui entoure le parasite. Le processus gagne ensuite le foie et la lèpre peut se généraliser. Le fait en lui-même est intéressant, car il fait ressortir un mode de propagation de la lèpre murine, sans doute rare, mais certain. Un parasite qui effectue des migrations à partir de l'intestin peut amener un virus déterminé, le bacille de la lèpre contenu dans cet intestin et le localiser dans le foie. C'est ce qui a fait dire au professeur Borrel et à moi-même que la larve du Tænia pouvait peut-être en même temps transporter et localiser le virus inconnu du sarcome.

Personnellement, j'ai été frappé par le fait suivant : sur cinq cas de sarcome, dent j'ai pu faire l'autopsie et sur lesquels j'ai pratiqué des coupes, il a été possible de trouver quatre fois le bacille de Stephansky dans la membrane. Les rats avaient été divisés en deux lots, le premier avait ingéré en même temps des œufs de Tænia crassicollis et des bacilles de Stephansky, le second lot uniquement des œufs de Tænia. Manifestement, les sarcomes se sont produits de préférence dans le premier lot, et ceci nous amène à faire les constatations suivantes :

Les cellules lépreuses, bourrées de bacilles, se disposent concentriquement dans la membrane, tout autour du parasite ; ce dernier élimine évidemment des toxines, des poisons qui entrent en contact avec les cellules lépreuses. Ceci ne nous paraît nullement être une hypothèse. Les bacilles lépreux sont constitués, en grande partie, par des cires. Or, une des propriétés des cires est de fixer, par adsorption, des toxines ; c'est une notion classique. Il est donc possible de concevoir qu'en un endroit déterminé de la membrane il se fait une accumulation de plus en plus forte de toxines. Cette fixation de toxines sur place peut-elle avoir, par action catalytique, une influence sur certaines cellules avoisinantes? Cela est possible, mais reste encore dans le domaine de l'hypothèse.

Frappé par ces constatations, j'ai recherché la présence des bacilles lépreux dans tous les cas de sarcome du foie du rat que j'ai pu examiner. Je n'ai pu les retrouver, l'association de la lèpre et

(1) Borrel (A.) et Larrousse (F.). - Localisation lépreuse dans le foie du rat par le cysticerque du Tænia crassicollis. C. $R$. Soc. biol., CV, 1930, p. 822, et Bull. assoc. franç. étud. du cancer, XXIX, 1930, $\mathrm{n}^{\circ} 9$, p. 1. 
du sarcome n'est donc pas obligatoire ; la lèpre paraît seulement dans certains cas, favoriser l'apparition du sarcome.

En examinant plus en détail ces cas d'association de lèpre et de sarcome chez le rat, on est, en premier lieu, frappé de la disposition des cellules lépreuses primitives dans la membrane; elles se disposent suivant un plan, le fait est certain, et nous retrouvons ici la conception du professeur Borrel, le plan neuro-tropho-pigmentaire (Pl. X, fig. 1). C'est dans ce plan que se disposent les cellules lépreuses, qui deviennent ainsi des cellules réceptrices, sur lesquelles Borrel a toujours insisté. La cellule lépreuse, dans le cas présent, n'est pas spécifique; elle réunit seulement les propriétés de toute cellule réceptrice. Pour ces raisons, je crois que l'on peut actuellement, en présence d'un élevage de rats réfractaires au sarcome malgré l'ingestion de nombreux œufs de Tænia crassicollis, rendre l'apparition de ce-sarcome possible en faisant ingérer, en même temps que les œufs, des fragments de tissus de rats lépreux riches en bacilles. L'expérimentateur devient maître d'une cellule réceptrice et peut la diriger, grâce au parasite, en bonne place dans le plan neuro-tropho-pigmentaire.

Dans la grande majorité des cas de sarcome du foie du rat, la lèpre n'existe pas; cela est un fait évident. J'ai voulu rechercher si, dans ces cas, il n'était pas possible de mettre en relief d'autres cellules réceptrices. Nous retrouvons ici la cellule pigmentaire, notion fondamentale sur laquelle a toujours insisté également le professeur Borrel. La fig. 2 (Pl. X) montre la disposition des cellules pigmentaires suivant le même plan que les cellules lépreuses. J'ai fait l'étude de ce pigment, il s'agit d'un pigment ferrugineux, hémosidérine ou pigment très rapproché de ce dernier. Il est, sans doute, associé à un complexe lipo-protéïque, la réaction du bleu de Nil étant légèrement positive après passage de la coupe dans l'eau oxygénée. Ces cellules pigmentaires peuvent, à mon avis, soit fixer un virus, soit fixer des toxines; il est égale-

Explication de la Planche $\mathrm{X}$

Cellules réceptrices

Fig. 1. - Cellules pigmentaires.

Fig. 2. - Cellules lépreuses. 


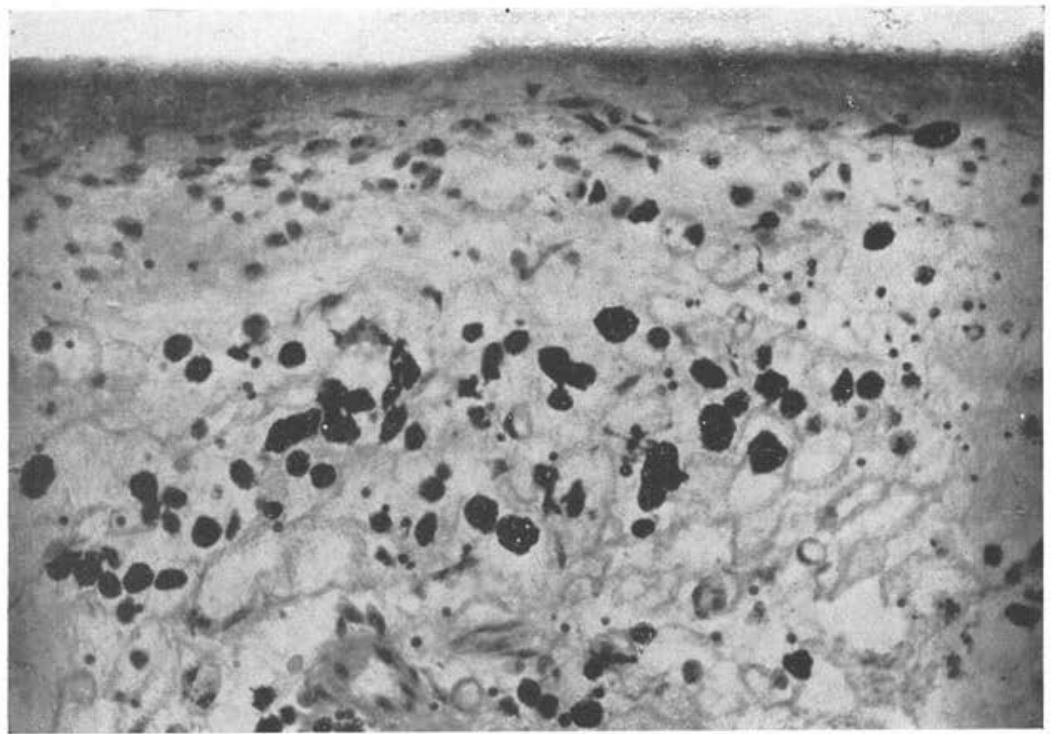

FIG. 1

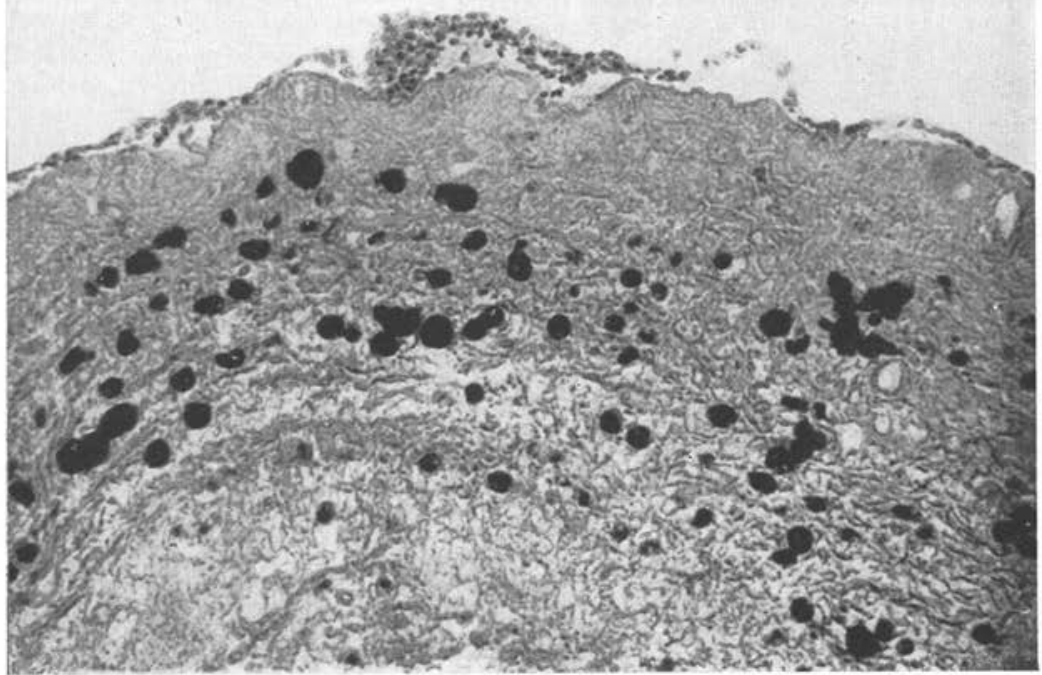




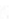


ment de notion classique que les sels de certains métaux peuvent précipiter les toxines. On retomberait ainsi dans le cas précédent de la lèpre. En un endroit déterminé de la membrane (dans le plan), il peut y avoir précipitation et concentration de toxines.

Je ne veux pas insister davantage sur ces faits et me prononcer définitivemeñt. Je montre simplement qu'en dehors du parasite Cysticercus fasciolaris, qui est obligatoire pour la production du sarcome, il est possible de mettre en évidence, dans la membrane de réaction de l'hôte, des cellules réceptrices capables de fixer soit un virus, soit des toxines ou poisons éliminés par le ver. Les constatations fäites avec les cellules lépreuses sont très démonstratives, car on retrouve la lèpre quel que scit le stade du sarcome. Pour les cellules pigmentaires, cela est plus délicat et, à mon avis, on ne peut les rechercher avec profit que dans les sarcomes au début. Le pigment étant un produit de déchet peut s'éliminer et, dans les cas avancés, on ne retrouve plus cette disposition concentrique si curieuse.

Des rats porteurs de cysticerques font du sarcome ou n'en font pas, suivant qu'ils ont ou qu'ils n'ont pas de cellules réceptrices. Cette notion peut-elle se concilier avec la notion d'hérédité mise en avant dans la génèse de ces sarcomes? Peut-être oui, si on envisage le processus héréditaire dans le seul cadre de l'hérédité pigmentaire.

Faculte de médecine de Strasbourg. 